\title{
Trajectory Control of Designed Experimental Mobile Robot
}

\author{
Şahin YILDIRIM \\ Department of Mechatronics Engineering \\ Erciyes University \\ Kayseri, Turkey \\ sahiny@erciyes.edu.tr
}

\author{
Berkay EREN \\ Department of Mechatronics Engineering \\ IskenderunTechnical University \\ Hatay, Turkey \\ berkay.eren@iste.edu.tr
}

\begin{abstract}
Due to advancing technology; nowadays mobile robot applications in hospitals have been increased. For that reason, it is very important and necessary to analyze the trajectory of such helping robotic system. However; there are many types of mobile robots have been utilized in hospital applications such as helping nurses. In this simulation study; a designed and controlled mobile robot was controlled by using standard feedback controllers. On the other hand, the robot was also tested with disturbances of ground surface roughness. The simulation results showed that the standard PID controller has superior performance to overcome the surface roughness of the robot trajectory compared to the $P$ and $P D$ controller. In addition, this robot is in a better position in terms of safety as it has the ability to distinguish patients and it costs less than robots of similar construction.
\end{abstract}

Keywords - Mobile robot, trajectory control, PID controller, stability, feeding system

\section{INTRODUCTION}

Mobile robots have become an essential component of automation systems since they are recently involved in many aspects of industrial applications or everyday life. Mobile robots can be divided into two groups according to the elements that provide movement, mobile robots with wheels and legged mobile robots which are inspired by some animal gait structures [1]. Studies on mobile robots are similar in the two groups. It can be classified as mapping and localization, trajectory analysis, determining the most suitable way to target with optimization algorithms, torque and speed control, avoiding static and/or dynamic obstacles [2]. Most mobile robots have differential transmissions. This situation limits the mobility of the robot since its orientation must always change according to the direction of movement. A mobile robot with omnidirectional wheels can perform movements in any direction without the need for redirection. In other words, because this type of wheels provides more free movement by increasing the degree of freedom, omnidirectional wheels are used in the designed and produced the system $[3,4]$.

In the studies conducted in the literature, many control methods such as PID control, fuzzy logic, artificial neural networks, fuzzy-artificial neural network, genetic algorithmbased PID, fuzzy PID were used for the problem of tracing the trajectory. But the most commonly used controller is PID and different controllers based PID controllers. Campos et al. [5] have presented an adaptive system using the particle swarm optimization (PSO) technique to set parameters of the PID controller.
While the errors in linear and angular velocities were the inputs of the fuzzy-PID controller, the membership functions were determined according to the results of the optimization. Conventional PID was compared to fuzzy-PID approaches. Mohamed and Abbas [6] have investigated the firefly algorithm to determine the parameters when designing a nonlinear fuzzy-PID controller for optimum trajectory tracking.

Abdalla et al. [7] have proposed the Takagi Sugeno type fuzzy logic controllers optimized by the PSO method. In this study, it was aimed to provide trajectory tracking control of the mobile robot using two optimized fuzzy controllers for speed control and azimuth control, while the results showed very good performance for the used controller. Mendili and Bouani [8] have tried the predictive control approach with three independent driving wheels based on kinematic and dynamic models of the mobile robot which has omni wheels. Two predictive controllers have been developed. The first was applied to the kinematic model and the second was based on the dynamic model. Two controllers have been compared, and studies have been performed to demonstrate the effectiveness of the predictive controller with kinematic and dynamic models. Demirbaş and Kalyoncu [9] have designed PID and kinematic based backstepping controller by obtaining a mathematical model of a non-holonomic mobile robot. They used PID controller for speed control of motors, backstepping controller for trajectory tracking. The performances of the controllers were tested by using Matlab / Simulink. Xu et al. [10] have designed a conventional PID and fuzzy-PID controller for a trajectory tracking problem of a mobile robot with differential driving and observed a $\% 40$ reduction in the amount of fuzzy-PID controller overflow. Omid et al. [11] have designed and implemented a new adaptive trajectory tracking controller for a non-holonomic wheeled mobile robot that has unknown parameters and indefinite dynamics. They have developed a backstepping controller which does not require knowledge about robot dynamics using artificial neural network. Yıldırım and Savaş [12], carried out trajectory analysis on Hemisson mobile robot using conventional PID and artificial neural network based adaptive $\mathrm{P}$ controller. 


\section{HAYAMOR (ASSISTANT MOBILE ROBOT FOR PATIENTS)}

Mobility of mobile robot designed and implemented for the purpose of helping patients was simplified for different surfaces such as rough, slippery using omnidirectional wheels. On the other hand, the reason for the use of 3 wheels is to reduce balance and vibration problems. The proposed, designed and produced mobile robot structure is shown in Figure 1. The proposed mobile robot system consists of $5 \mathrm{DC}$ motor, 3 stepper motor were employed to move the body of the robot on the surface. One motor is used to supply water and pills to patients with the turning movement. The other one is utilized for a sliding top mechanism which is used for the emergency of hands. The system is actuated by motors energized by two $12 \mathrm{~V}$ battery. The Arduino Mega is used as a controller which controls the wheel motor speeds and the motor speeds used to drive the drug feeding mechanism and lid.

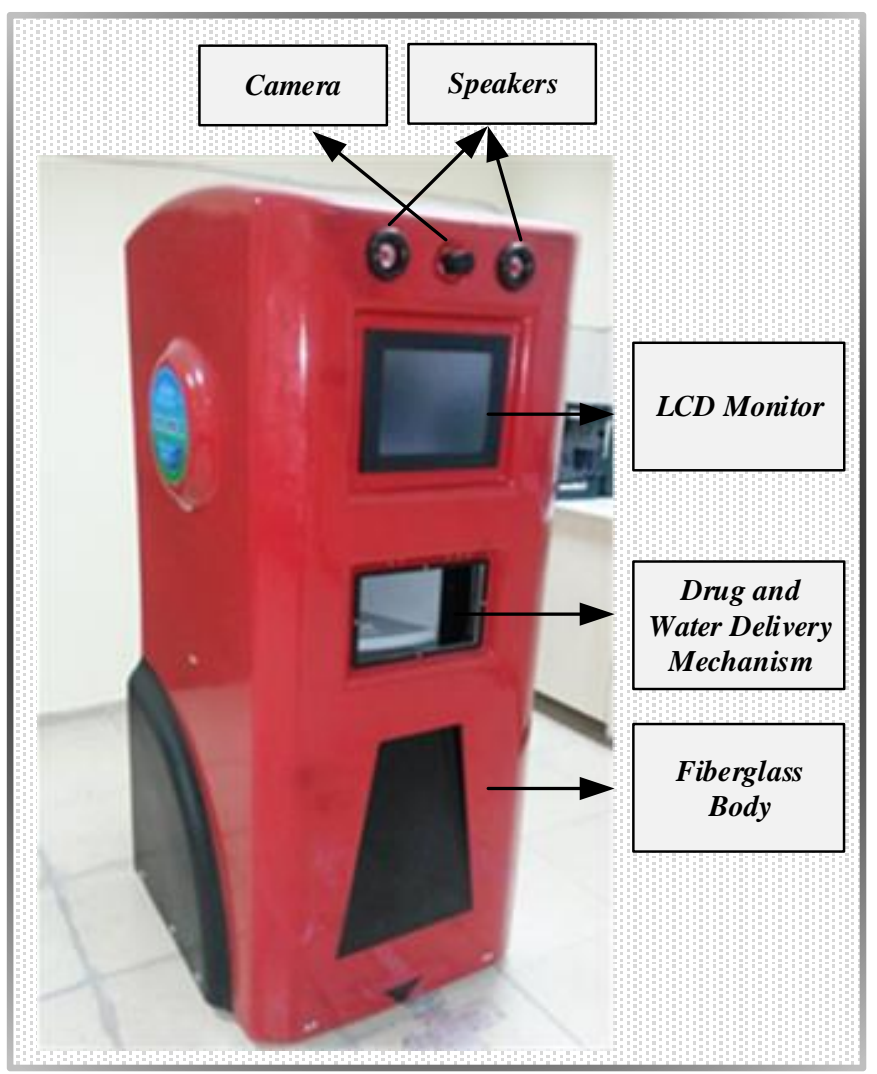

Fig. 1. Designed and controlled mobile robot

\section{A. Kinematics of Proposed Mobile Robot}

The kinematics of the designed and applied mobile robot consists of three Swedish wheels, which are mounted symmetrically with a 120-degree angle to each other. Each wheel, which is driven by a DC stepper motor, is at a distance $\mathrm{L}$ to robot's center of rotation R. (see Fig 2.)

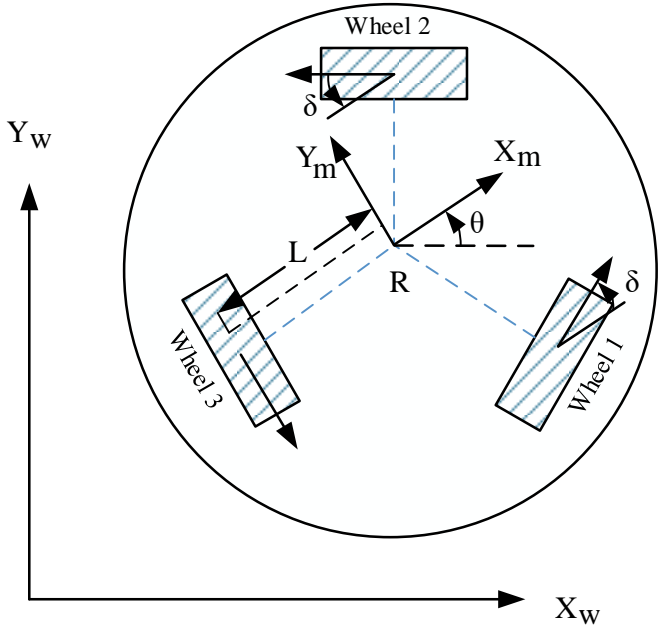

Fig. 2. Schematic model of mobile robot wheel mechanism

As shown in Figure 2, $\left[\mathrm{X}_{\mathrm{w}}, \mathrm{Y}_{\mathrm{w}}\right]$ refers to the global coordinate system, while $\left[\mathrm{X}_{\mathrm{m}}, \mathrm{Y}_{\mathrm{m}}\right]$ refers to the coordinate system of the mobile robot when moving. The transformation matrix which provides transformation from robot coordinate system to global coordinate system is in equation 1 ,

$$
{ }^{w} R_{m}=\left[\begin{array}{cc}
\cos \theta & -\sin \theta \\
\sin \theta & \cos \theta
\end{array}\right]
$$

The inverse kinematic equations of the mobile robot are given in equation $2,3,4 . \mathrm{V}_{\mathrm{i}}, \mathrm{i}=1,2,3$ denotes the linear velocity of each wheel.

$$
\begin{gathered}
V_{1}=\sin \left(\frac{\pi}{3}-\theta\right) \cdot \dot{x}+\cos \left(\frac{\pi}{3}-\theta\right) \cdot \dot{y}+L \dot{\theta} \\
V_{2}=-\sin \left(\frac{\pi}{3}+\theta\right) \cdot \dot{x}+\cos \left(\frac{\pi}{3}+\theta\right) \cdot \dot{y}+L \dot{\theta} \\
V_{3}=\sin (\theta) \cdot \dot{x}-\cos (\theta) \cdot \dot{y}+L \dot{\theta}
\end{gathered}
$$

The angle $\theta$ indicates the direction of the robot according to the global coordinate system. $\mathrm{w}_{1}, \mathrm{w}_{2}, \mathrm{w}_{3}$ indicate the angular velocity of the wheels, vector $\left[\begin{array}{lll}\dot{x} & \dot{y} & \dot{\theta}\end{array}\right]^{T}$ defines linear velocity of the robot in the $\mathrm{X}$ direction, $\mathrm{Y}$ direction and angular velocity in the global coordinate system, respectively. If the equations in Equation 2,3,4 are reorganized in the vectormatrix form, Equation 5 is obtained.

$$
\left[\begin{array}{l}
V_{1} \\
V_{2} \\
V_{3}
\end{array}\right]=\left[\begin{array}{l}
r w_{1} \\
r w_{2} \\
r w_{3}
\end{array}\right]=P(\theta)\left[\begin{array}{c}
\dot{x} \\
\dot{y} \\
\dot{\theta}
\end{array}\right]
$$

$\mathrm{V}_{1}, \mathrm{~V}_{2}, \mathrm{~V}_{3}$ indicate the linear speeds of the wheels. The Equation 5 can be displayed in a different version as in Equation 6.

$$
\left[\begin{array}{c}
\dot{x} \\
\dot{y} \\
\dot{\theta}
\end{array}\right]=P^{-1}(\theta)\left[\begin{array}{l}
V_{1} \\
V_{2} \\
V_{3}
\end{array}\right]
$$


When the Equation 6 was solved, the reverse of the $\mathrm{P}$ matrix can be found.

$$
\left[\begin{array}{ccc}
P^{-1}(\theta)= \\
\frac{2}{3} \cos (\delta+\theta) & -\frac{2}{3} \cos (\theta-\delta) & \frac{2}{3} \sin \theta \\
\frac{2}{3} \sin (\delta+\theta) & -\frac{2}{3} \sin (\theta-\delta) & -\frac{2}{3} \cos \theta \\
\frac{1}{3 L} & \frac{1}{3 L} & \frac{1}{3 L}
\end{array}\right]
$$

The angle $\delta$ indicates the orientation of the wheels according to the robot coordinate system and is equal to 30 degree [13]. At the same time, the forward kinematic equations of the system are reached with equation 7 . The wheel radius $r$ is 0.102 meters and the distance of the wheel centers to the robot center of rotation is 0.315 meters.

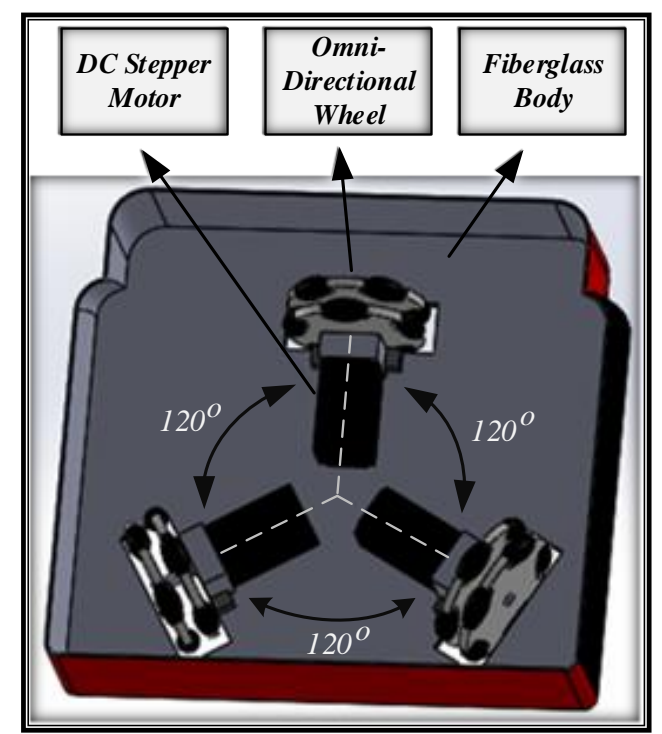

Fig. 3. Wheel structure of proposed mobile robot system and view of drive elements.

\section{THE PROPOSED CONTROL SYSTEM}

The use of standard feedback controllers is simpler and easier to implement.

In this study, the system trajectory was controlled with standard P, PD and PID control organs.

\section{A. Standard Feedback Controllers}

This section describes standard feedback controllers such as P, PD, PID controllers. Proportional-integral-derivative is the control method which can be used in the control of nonlinear systems as well as control of linear systems which has a wide usage area in modern industry. On the other hand; it is a control effect that combines the advantages of three basic control effects into one unit. Nevertheless, the steady state error that can occur is reset with the integral effect, the stability and response rate of the derivative effect system is increased [14]. The PID control method is an effective and the most preferred control structure that combines the advantages of the control effects P, PI and PD which have a zero steady state error in the system [15]. The mathematical equation of PID is generally given in Equation 8.

$$
u(t)=K_{p} e(t)+K_{i} \int_{0}^{t} e(t) d t+K_{d} \frac{d e}{d t}
$$

Here, the $r(t)$ input (reference) signal, $u(t)$ control signal, $e(t)$ error signal and $y(t)$ is the output of the system. $K_{p}, K_{i}, K_{d}$ coefficients are proportional, integral and derivative coefficients.

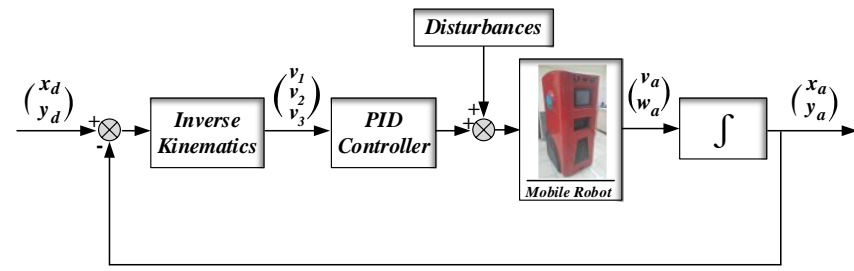

Fig. 4. Proposed mobile robot's closed loop block diagram with PID controller

The block diagram given in figure $4,\left[\mathrm{x}_{\mathrm{d}}, \mathrm{y}_{\mathrm{d}}\right]$ defines the desired trajectory in the $\mathrm{X}$ and $\mathrm{Y}$ axis, while $\left[\mathrm{v}_{\mathrm{a}}, \mathrm{w}_{\mathrm{a}}\right]$ signifies to the mobile robot's actual linear and angular velocity. $\left[\mathrm{x}_{\mathrm{a}}\right.$, $\mathrm{y}_{\mathrm{a}}$ ] represents the actual position of the mobile robot.

\section{SIMULATION RESULTS}

In all simulation studies performed for six reference trajectories, these values were taken as control effect. In this control work, the linear velocity of the robot was $0.055 \mathrm{~m} / \mathrm{s}$ and the angular velocity was taken as $\mathrm{pi} / 20 \mathrm{rad} / \mathrm{s}$ (9 degrees $/ \mathrm{s})$. In the Simulink blocks added to the system, power of the noise effect (band-limited white noise) was taken as 0.1 , the sampling time was 1 second and the same values were applied for all trajectories.

Mobile robot trajectories which are gone six different rooms to give patients' drugs have been performed with P, PD, PID controller in simulation environment. In the simulation study, while setting the PID parameters, each parameter is increased starting from very small values. At the end of each trial, it was optimally adjusted after many trials, taking into account the conditions such as the steady state error approaching zero and the mobile robot not swinging The optimum PID parameters found are presented in Table 1.

\begin{tabular}{|c|c|c|c|}
\hline & $\mathbf{K}_{\mathbf{P}}$ & $\mathbf{K}_{\mathbf{i}}$ & $\mathbf{K}_{\mathbf{d}}$ \\
\hline $\mathbf{P}$ & 33 & - & - \\
\hline PD & 65 & - & 5 \\
\hline PID & 12 & 51 & 0.04 \\
\hline
\end{tabular}

\section{TABLE I. GAIN PARAMETERS OF CONTROLLERS}




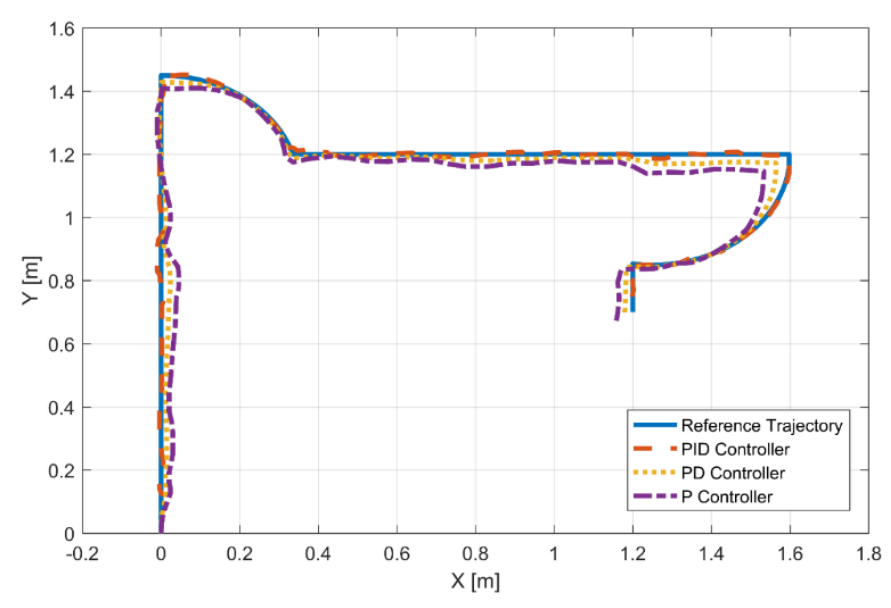

Fig. 5. Trajectory change of robot from the starting point to station $1($ Time $=71.2 \mathrm{~s})$

As shown in Figure 5, it was seen reference trajectory and trajectory changing controlled by various controllers. While the PID controlled robot can be said to have a sufficient follow-up performance on the first linear pathway, it is observed that it has not deviated from the follow-up too many times in the second linear path. In the first turn, starting and finishing the turn follow-up performance was sufficient, the reference trajectory was followed in the last turn with little error. In the trajectory controlled with the PD controller, it did not oscillate in the rotation part, but it did not catch the reference very well on the linear and rotation road. In general, the P controller could not achieve the desired success in all trajectory traces, and problems such as high error and inability to approach the reference trajectory were observed.

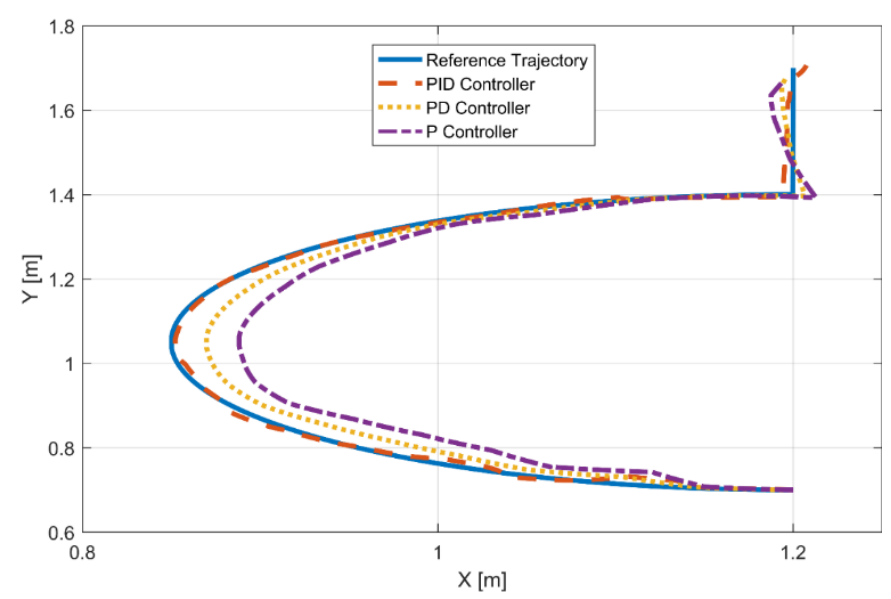

Fig. 6. Trajectory change of robot from the station 1 to station $2($ Time $=25.45 \mathrm{~s})$

Figures 6 and 8 show the same trajectory and control performance. This is because the same trajectories were tracked in both graphs and the results were the same because the noise value and controller parameters used were the same, but their position in the global coordinate system is different. Based on the performance of the PID controller, the system's follow-up performance fluctuates after the start of the rotation, while the second half gives better results. Good results were obtained, even though there were small deviations in the linear path following the rotation and in the following.

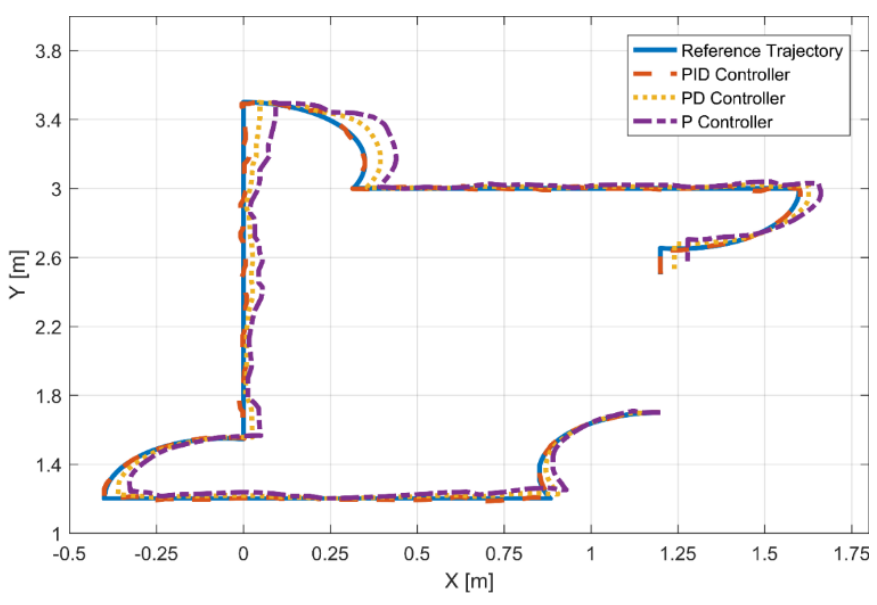

Fig. 7. Trajectory change of robot from the station 2 to station 3 (Time $=$ $132.4 \mathrm{~s})$

The trajectory in Figure 7, which has the longest path and time, summarizes the follow-up performance of the controllers in general. As in previous trajectories, the PID controller exhibited a sufficient but not very good control performance on linear paths, while the error occurred at some moments around $2-3 \mathrm{~cm}$. When the first and last rotation ability was examined, the trajectory was tracked well. The PD controller did not oscillate much, but it was observed that it could not reach the reference and there was a permanent error.

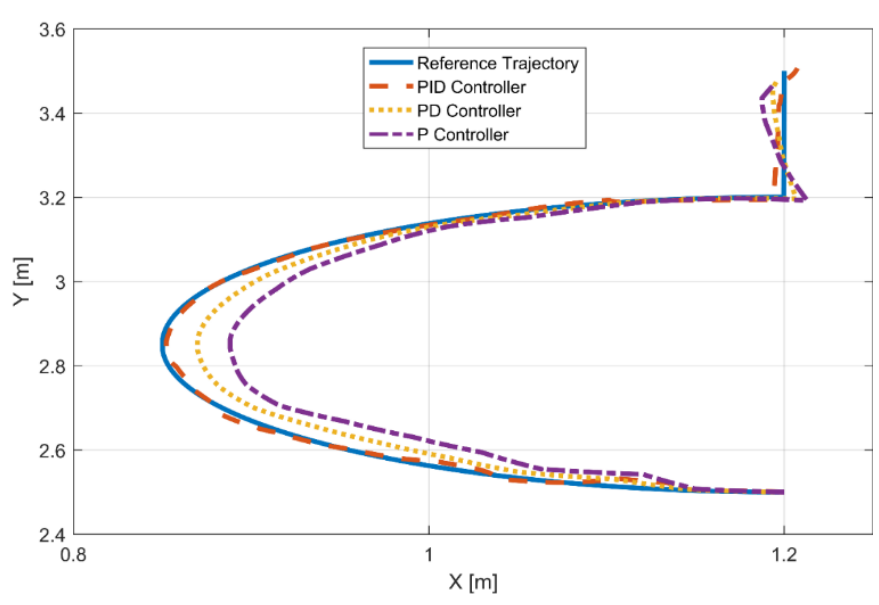

Fig. 8. Trajectory change of robot from the station 3 to station 4 (Time $=$ $25.45 \mathrm{~s})$

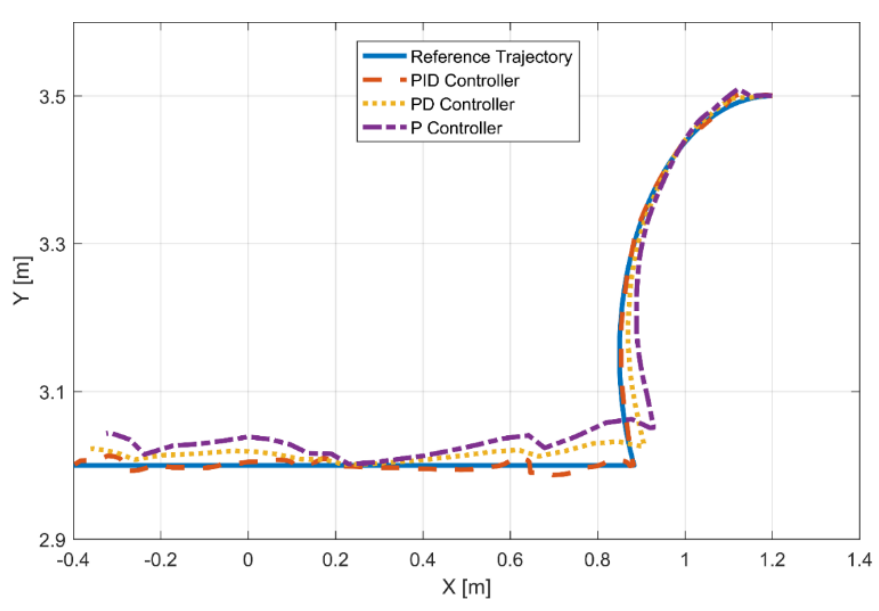

Fig. 9. Trajectory change of robot from the station 4 to station 5 (Time $=$ $36.15 \mathrm{~s})$ 
In Figure 9, the rotation performance for the PID controller is very good except for the start of the turn. The resulting error is in millimeter levels. Linear performance is not sufficient levels expected.

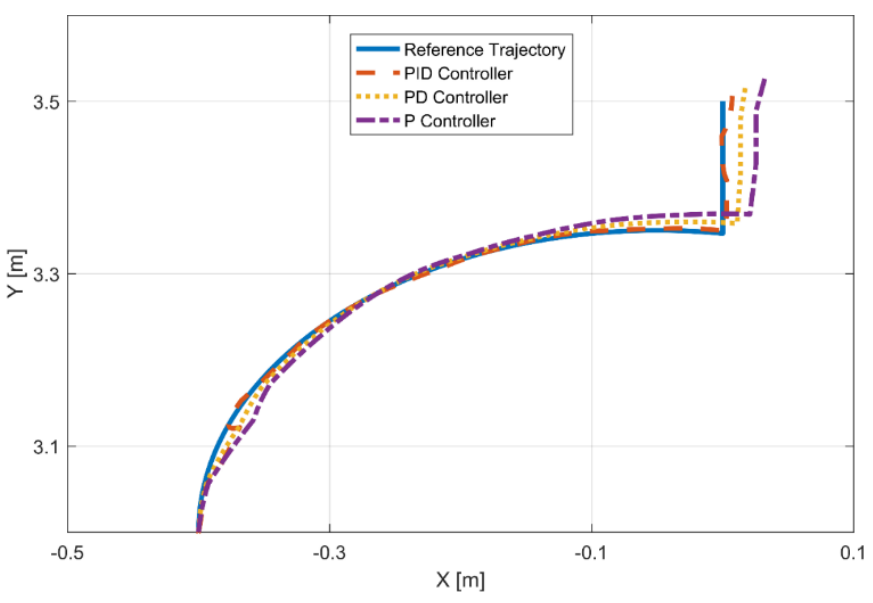

Fig. 10. Trajectory change of robot from the station 5 to station 6 (Time $=$ $13.7 \mathrm{~s})$

In Figure 10, where the last trajectory is indicated, a good return performance for all controllers can be mentioned, while the best response to the sudden change of direction after the turn has given PID controller.

\section{CONCLUSIONS AND DISCUSSIONS}

This paper has presented a proposed mobile robot trajectory control with standard feedback controllers. The prescribed trajectories were set to six feeding stations for patients. As can be seen from graphs; the trajectories represent linear and non-linear behaviour because of waiting stations conditions. The moving surface of the robot consists of some disturbances such as small surface roughness. In terms of these roughnesses; the robot has difficulty to follow the desired trajectory without small errors.

The standard feedback controllers such as P, PD, PID were used to control the robot's prescribed trajectory with disturbances. There is also a big effort of the robot by weights of batteries.

The proposed robot's prescribed trajectory was controlled with small errors by the PID controller. Other controllers have poor performance rather than PID controller.

The production cost of this robot, which has been produced, is lower compared to its equivalents. However, if mass production is started, it will have a lower cost than its current cost. The most important feature that distinguishes this robot from other hospital robots is that it can separate the patients and provide medicine to the patients without causing any confusion, thanks to the camera systems on it.

\section{REFERENCES}

[1] Siegwart, R. Roland, R. et al. "Introduction to autonomous mobile robots". MIT Press, 2011

[2] Sariff, N., and Norlida Buniyamin. "An overview of autonomous mobile robot path planning algorithms." 2006 4th student conference on research and development. IEEE, 2006.

[3] Watanabe, Keigo. "Control of an omnidirectional mobile robot." 1998 Second International Conference. Knowledge-Based Intelligent Electronic Systems. Proceedings KES'98 (Cat. No. 98EX111). Vol. 1. IEEE, 1998.

[4] Hacene, Nacer, and Boubekeur Mendil. "Fuzzy behavior-based control of three wheeled omnidirectional mobile robot." International Journal of Automation and Computing 16.2 (2019): 163-185.

[5] Campos, J., Jaramillo, S., Morales, L., Camacho, O., Chávez, D., \& Pozo, D. "PSO Tuning for Fuzzy PD+ I Controller Applied to a Mobile Robot Trajectory Control". International Conference on Information Systems and Computer Science (INCISCOS) pp.62-68, 2018.

[6] M.J. Mohamed and M.Y. Abbas, "Design a Fuzzy PID Controller for Trajectory Tracking of Mobile Robot," Engineering and Technology Journal, Vol. 36, Part A, No. 1, pp. 100-110, 2018.

[7] T.Y. Abdalla and A.A. Abdulkareem, "PSO Optimized Fuzzy Control Scheme for Mobile Robot Path Tracking", International Journal of Computer Applications, Volume 76- No.2, pp. 0975 - 8887, 2013.

[8] Mendili, M., \& Bouani, F. "Predictive Control of Mobile Robot Using Kinematic and Dynamic Models". Journal of Control Science and Engineering, 2017.

[9] F. Demirbas and M. Kalyoncu, "Differential drive mobile trajectory tracking with using PI and kinematic based backstepping controller", Sujest, vol. 5, no. 1, pp. 1-15, 2017.

[10] Xu, Q., Kan, J., Chen, S., \& Yan, S. "Fuzzy PID based trajectory tracking control of mobile robot and its simulation in Simulink". International journal of control and automation, 7(8), pp.233-244, 2014.

[11] Mohareri, O., Dhaouadi, R., \& Rad, A. B. "Indirect adaptive tracking control of a nonholonomic mobile robot via neural networks". Neurocomputing, 88, pp.54-66, 2012.

[12] Yildirim Ş., Savaş S., "Trajectory control of a mobile robot using neural network for frail blind persons", International Review of Applied Sciences and Engineering, vol.4, pp.27-34, 2013.

[13] Li, Xiang, and Andreas Zell. "Motion control of an omnidirectional mobile robot." Informatics in Control, Automation and Robotics. Springer, Berlin, Heidelberg, pp. 181-193, 2009.

[14] Johnson, Michael A., and Mohammad H. Moradi. PID control. Springer-Verlag London Limited, 2005.

[15] Li, Yun, Kiam Heong Ang, and Gregory CY Chong. "PID control system analysis and design." IEEE Control Systems Magazine 26.1: 32-41, 2006. 
